\title{
Solution for intra/inter-cluster event-reporting problem in cluster-based protocols for wireless sensor networks
}

\author{
Raed Taleb Al-Zubi ${ }^{1}$, Abdulraheem Ahmed Kreishan ${ }^{1}$, Mohammad Qasem Alawad ${ }^{1}$, \\ Khalid Ahmad Darabkh ${ }^{2}$ \\ ${ }^{1}$ Department of Electrical Engineering, School of Engineering, The University of Jordan, Amman, Jordan \\ ${ }^{2}$ Department of Computer Engineering, School of Engineering, The University of Jordan, Amman, Jordan
}

\begin{tabular}{l} 
Article Info \\
\hline Article history: \\
Received Feb 17, 2021 \\
Revised Sep 3, 2021 \\
Accepted Sep 15, 2021
\end{tabular}

Keywords:

Cluster-based networks

Routing protocols

SIICERP

Wireless sensor networks

\begin{abstract}
In recent years, wireless sensor networks (WSNs) have been considered one of the important topics for researchers due to their wide applications in our life. Several researches have been conducted to improve WSNs performance and solve their issues. One of these issues is the energy limitation in WSNs since the source of energy in most WSNs is the battery. Accordingly, various protocols and techniques have been proposed with the intention of reducing power consumption of WSNs and lengthen their lifetime. Cluster-oriented routing protocols are one of the most effective categories of these protocols. In this article, we consider a major issue affecting the performance of this category of protocols, which we call the intra/inter-cluster event-reporting problem (IICERP). We demonstrate that IICERP severely reduces the performance of a cluster-oriented routing protocol, so we suggest an effective Solution for IICERP (SIICERP). To assess SIICERP's performance, comprehensive simulations were performed to demonstrate the performance of several cluster-oriented protocols without and with SIICERP. Simulation results revealed that SIICERP substantially increases the performance of cluster-oriented routing protocols.
\end{abstract}

This is an open access article under the $\underline{C C B Y-S A}$ license.

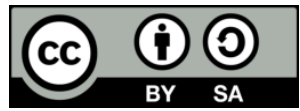

\section{Corresponding Author:}

Khalid Ahmad Darabkh

Department of Computer Engineering, School of Engineering, The University of Jordan

P.O. Box, Amman 11942, Jordan

Email:k.darabkeh@ju.edu.jo

\section{INTRODUCTION}

Mechanical-systems (MEMS) technology, and digital electronic switches, the implementation of WSNs in the real world become feasible. In the last few years, wireless sensor networks (WSNs) have been considered as a hot research topic with a rapidly increasing set of important applications. A WSN is made up of a considerable number of small, intelligent devices termed sensor nodes. The sensor nodes differ in their characteristics such as cost, power consumption, and size. Each sensor node consists of a power unit which is basically a small battery, communication unit which contains a wireless transceiver, sensing unit which contains an analog to digital converter (ADC), and data processing unit which contains a small processor and a limited size storage unit [1]-[4].

Sensor nodes have different sensing capabilities based on the environment. They sense the environment, process the data, and transmit it to the receiver via wireless channel. These nodes are randomly deployed in distant regions for monitoring aims. In a WSN, the primary mission of deployed sensor nodes is to gather the data from the area of interest region and then direct it toward the sink node or base station (BS) by means of pre-determined channels. The BS then transmits these data via wireless channel to a remote destination for further analysis and processing. BS may be inside the region of sensing or far from it according 
to the application area [5]. There are various kinds of sensors as radar sensors, infrared sensors, visual and magnetic sensors, acoustic sensors, thermal sensors. Accordingly, WSN can be used in different applications such as healthcare applications, environmental applications, healthcare applications, and many other fields [6], [7]. Although the WSNs have many interesting applications and attractive characteristics, they face many challenges such as energy constraint, node deployment, data aggregation, network heterogeneity, scalability issue, and security [8]-[14]. Between those challenges, energy hindrance is the most worrying matter for several researchers in domain of WSNs. Sensor nodes need a lot of energy since they sense, process, transmit and collect data. In fact, the battery is the main source of energy in these nodes, and it is nearly impossible to change or recharge it after the nodes have been deployed in the network [10]-[13]. As a result, well-investigated protocols for collecting data in the wireless network are required in order to reduce the consumption of power and hence extend the network's lifetime.

Numerous energy-efficient data collection techniques were proposed for WSNs, such as routing protocols. These protocols can be categorized into three major groups: tree-oriented routing protocols [1], [2], flat-oriented routing protocols [3]-[5], and cluster-oriented routing protocols [6]-[24]. In this article, we principally focus on the group of cluster-oriented routing protocols, which has demonstrated to be on the top of energy-saving routing protocols [25]-[32]. In cluster-oriented routing protocols, the wireless network is organized into groups, which are named as clusters. Each cluster has one cluster head $(\mathrm{CH})$ and other normal nodes named as member nodes. All member nodes are in charge of sensing the data and transmitting it to the $\mathrm{CH}$, while the $\mathrm{CH}$ is in charge of collecting data from member nodes, aggregating the data, and then transmitting it to the BS. It is noteworthy that the clustering in WSN can be either static or variable. In static clustering, clusters are usually formed once the nodes are deployed, and remain the same until the lifetime of the network expires. The role of $\mathrm{CH}$ in each cluster rotates among all nodes. On the other hand, in variable clustering, the network's lifetime is divided into numerous rounds. At the start of each round, new clusters are formed. There are some protocols which employ a third form of nodes known as relay nodes (RNs), which are in charge of receiving data from CHs and relaying it to the BS. Actually, WSNs can save energy by using cluster-based routing protocols, thus extending their lifespan. Figure 1 shows the architecture of cluster-based routing protocols.

All cluster-oriented routing protocols, whether static or variable, time driven or event driven, centralized or distributed protocols, have a major problem that significantly depletes nodes' batteries. This problem is known as the intra/inter-cluster event-reporting problem (IICERP). This matter manifests itself on two categories: intra-transmission and inter-transmission. If an event occurs in a certain network region during the first category, several nodes from the same cluster or dissimilar clusters will report the occurrence by sending identical data packets to their respective $\mathrm{CHs}$. In the second category, if an event occurs in a specified network region, many $\mathrm{CHs}$ will report the occurrence to the BS by sending identical data packets.

Figure 2 illustrates IICERP problem on both levels. The figure shows that nodes N1, N2, and N3 send comparable data packets to respective $\mathrm{CH}$ node $(\mathrm{CH} 1)$ to report on occurrence. Furthermore, it demonstrates that $\mathrm{CH} 1$ and $\mathrm{CH} 2$ send the $\mathrm{BS}$ an overly similar data packet to tell about event $\mathrm{B}$. Thus, reporting on event $\mathrm{A}$ necessitates the transmission of three identical data packets from three different nodes. As a result, $\mathrm{CH} 1$ will execute data aggregation for the same three data packets, thereby consuming more energy. Furthermore, transferring the same data packets to the $\mathrm{BS}$ by the $\mathrm{CH} 1$ and $\mathrm{CH} 2$ for reporting on the same event (event B) consumes an increasing amount of energy.

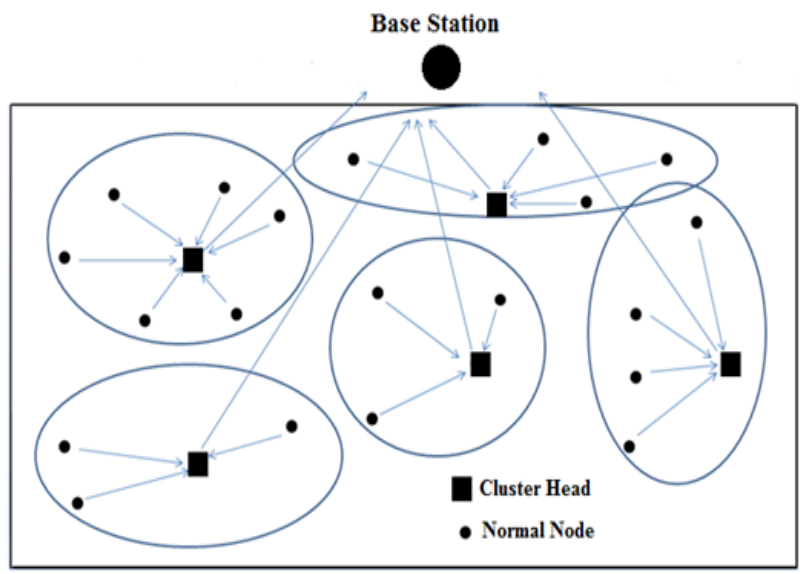

Figure 1. Architecture of cluster-based routing protocols 


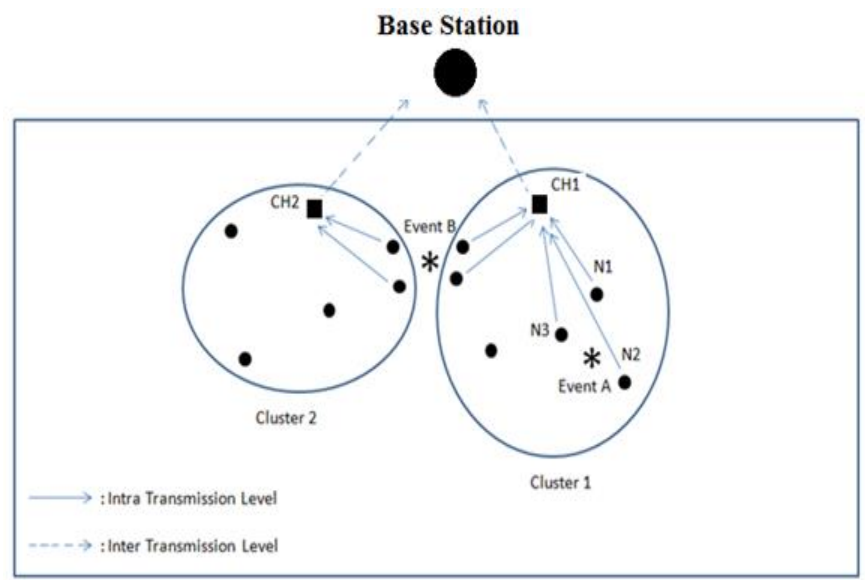

Figure 2. IICERP on both intra and inter transmission levels

In this research paper, we propose solution for intra/inter-cluster event-reporting problem (SIICERP), an effective Solution to the IICERP problem that enhances the performance of existing cluster-oriented routing protocols. In fact, SIICERP is absolutely consistent with cluster-oriented routing protocols and mainly aims to report an event through only one $\mathrm{CH}$ node and one normal node. We implement SIICERP into two clusteroriented routing protocols to assess its performance. The first employs a static clustering scheme, whereas the second employs a variable clustering scheme. The simulation findings reveal that SIICERP considerably conserves energy and thereby elongates the network's lifetime. It is worth mentioning that preliminary findings of this research have been published in [33].

The remainder of the paper is laid out as follows. Section 2 introduces the related work. SIICERP is presented and explained in section 3. In section 4, we utilize simulations to assess SIICERP's performance. Finally, concluding observations will be drawn in section 5.

\section{RELATED WORK}

In this work, we primarily introduce a solution for IICERP (SIICERP) in cluster-oriented routing protocols. In the literature, several cluster-based routing protocols have been developed [6]-[24], [34], [35]. However, in this work, we only consider two cluster-oriented protocols presented in [34] and [35] in order to demonstrate the influence of the suggested solution. In point of fact, we choose these two protocols because one is based upon the variable clustering mechanism [34] while the other relies on the static clustering mechanism [35].

Low energy adaptive clustering hierarchy (LEACH) is the first and most well-known cluster-oriented routing protocol proposed by the authors in [34]. This protocol divides the overall network lifetime into time slots named as rounds. Each round begins with a short setup period and ends with a long steady state period. In the setup period, $\mathrm{CH}$ nodes are chosen and clusters are established. The $\mathrm{CH}$ nodes are chosen in the form of a random probability. At the start of every single round, each node proceeds by generating a random number in the range from 0 to 1 . After then, the resulting number is compared to a preset limit $T(n)$ for the sake of determining whether this node is a normal node or a $\mathrm{CH}$ node. $T(n)$ is calculated using (1),

$$
T(n)=\left\{\begin{array}{cl}
0 & \text {,If } n \notin S \\
\frac{p}{1-p \times\left[r \bmod \left(\frac{1}{p}\right)\right]} & \text {,If } n \in S
\end{array}\right.
$$

where $r$ is the current round number, $n$ is the node, $p$ is the percentage of the desired number of $\mathrm{CH}$ nodes from all nodes in the network, and $S$ is the set of nodes that worked as $\mathrm{CHs}$ in the previous rounds.

Then, each $\mathrm{CH}$ node sends an announcement message to all nodes in the entire network, using carrier sensing multiple access (CSMA) protocol, informing them that it has been selected as $\mathrm{CH}$. Announcement message includes the location of selected $\mathrm{CH}$. Normal nodes will select their $\mathrm{CHs}$ on the basis of the strength 
of the signals of received messages. Each normal node sends a join message to the $\mathrm{CH}$ closest to it, informing it that it has joined the cluster. As a result, clusters are formed. The clusters, in general, have varying numbers of member nodes. All $\mathrm{CH}$ nodes produce time division multiple access (TDMA) schedules to arrange data transmission among member nodes in their clusters. They then broadcast these schedules to their member nodes, informing them of when each node should send the data it has collected. Each TDMA schedule is broken into time slots of equal length. The number of slots in the cluster is equal to the number of member nodes, and the length of every slot is the time each node takes to send its data packet. The setup period ends at this point, then the steady state period starts. The steady state period is divided into many time slots named frames. The total number of these frames in a cluster equals the total number of member nodes. In each cluster, member nodes send their data packet to their $\mathrm{CH}$ node in their own frame. After that, the $\mathrm{CH}$ aggregates these data packets along with its own data and sends the resulting data packet towards BS using CSMA protocol. Figure 3 shows the network lifetime according to the LEACH protocol.

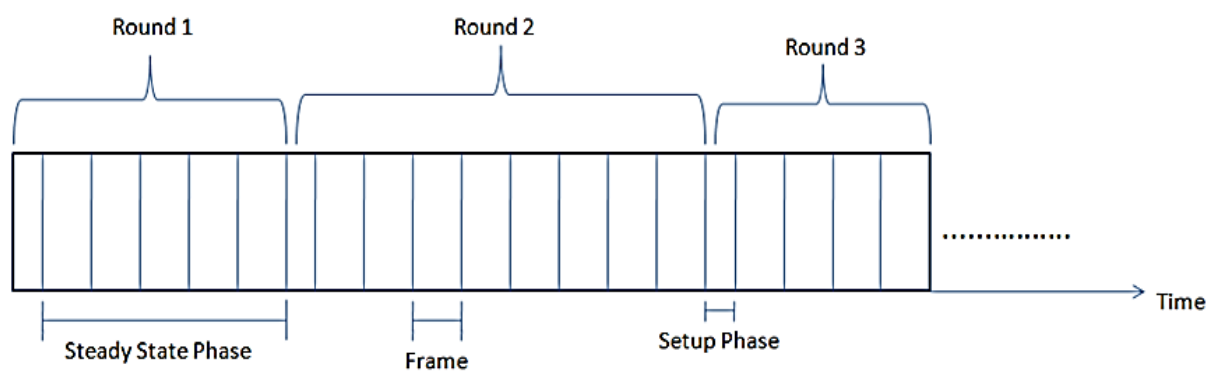

Figure 3. Network lifetime based on LEACH protocol

Load balancing cluster head ( $\mathrm{LBCH})$, an energy-saving routing protocol that uses a static clustering scheme, was proposed in [35]. In this protocol, each cluster has one $\mathrm{CH}$ node, member nodes, and one relay node (RN). The $\mathrm{CH}$ collects and aggregates data packets from its member nodes and sends them to the RN. The RN, in multi-hop routing, sends the data collected in each cluster to the BS. LBCH protocol divides the network lifetime into two periods: a short setup period and a long steady state period. At the setup period, BS broadcasts to all nodes in the network a hello message. The nodes therefore send their energy and locations to the BS. After that, the BS splits the network region into clusters of similar size. The width and length of each cluster do not exceed a predetermined threshold distance. Subsequently, the BS chooses one $\mathrm{CH}$ node and one $\mathrm{RN}$ for each cluster, that is, the initial CHs and RNs. The RN's role necessitates much energy because it receives the cluster's total data packets and then transmits these data packets to the next RN. Thus, the RNs are selected by computing the magnitudes of the nodes in each cluster to reduce the power consumption of these nodes. The magnitude of a node is inversely related to node's distance from the BS and linearly proportional to node's energy. The node with the greatest magnitude in the cluster will be chosen as the cluster's RN. The magnitude $M_{n}$ of node $n$ is calculated using (2),

$$
M_{n}=E_{n}+\frac{1}{d(n, B S)}
$$

where, $E_{n}$ refers to the node $n$ energy, and $d(n, B S)$ belongs to the distance from node $n$ to the BS. The BS determines the routing paths after selecting the RNs and sends the next RN hop to each RN. Also, the $\mathrm{CH}$ of each cluster is chosen by BS on the basis of the weight of each node. The weight $W_{n}$ of node $n$ is calculated using (3),

$$
W_{n}=E_{n}+\frac{1}{d(n, R N)}+\frac{1}{d_{n}}
$$

where $d(n, R N)$ belongs to the distance from node $n$ to the $\mathrm{RN}$, and $d_{n}$ represents the average distance from node $n$ to all other members in the cluster. The equation illustrates that the closest node to the RN and to the other members and which has the highest level of energy is chosen as $\mathrm{CH}$ for the cluster.

For the next rounds, the role of $\mathrm{CHs}$ and RNs is rotated between all nodes in the cluster, guaranteeing load balance among nodes. In this protocol, the nodes that were selected as $\mathrm{CHs}$ in the prior rounds will not be chosen as CHs in the present round, the same for RNs. More precisely, any node in the cluster may take the role of $\mathrm{CH}$ and $\mathrm{RN}$ only once in any round. Once all nodes in each cluster have taken their turn as $\mathrm{CH}$ and $\mathrm{RN}$, 
the same process will be repeated for choosing CHs and RNs. The BS then produces TDMA schedules for all cluster nodes with the aim of regulating the transfer of data among the nodes and the $\mathrm{CH}$. Furthermore, in order to regulate the transmission of data among each cluster and BS, the BS allocates each cluster a unique code division multiple access (CDMA) code. The steady state period is separated into identical frames named rounds whereas each round is separated into several time slots equal to the highest number of nodes in a cluster besides one more time slot designated for the purposes of controlling the next round. On the other hand, the member nodes send their own data packets to the respective $\mathrm{CH}$ in their allotted time slots. The $\mathrm{CH}$ then aggregates all data packets and forwards them to the respective RN in its cluster, which in turn sends them back to the BS in the multi-hop routing process. In the additional time slot, BS selects both new CHs and RNs for the next round and broadcasts a message about the selected CHs and RNs to all nodes before the next round begins.

\section{PROPOSED PROTOCOL: SIICERP}

\subsection{Design of SIICERP}

The idea behind SIICERP can be summarized as follows: if the sensing regions (i.e., approximated as circles around sensors [36]) of some sensors are overlapped, then similar packets will be redundantly sent by these sensors to report a common event occurs on the overlapped region. This idea is depicted in Figure 4. Based on this idea, SIICERP is designed such that a single packet is transmitted to report any event. The node that sends this packet is selected such that, in the network, the load is balanced and the total energy consumption is reduced. To satisfy these requirements, SIICERP is designed as follows:

a. First, SIICERP can work with any cluster-based scheme. During the setup time in each round, the clusters and their corresponding $\mathrm{CHs}$ are selected according to the used cluster-based scheme. The energy and the positions of the sensors in each cluster are sent to the BS via the CHs.

b. Second, to avoid interference, CDMA scheme is employed to conduct the communications between the BS and the clusters; different codes are assigned for different clusters and one code is used in one cluster.

c. Third, the BS runs a time-scheduling algorithm (discussed in section 3.2) to determine for each sensor the time slots in which the sensor can report an event and the time slots in which the sensor listens to its neighbors using their CDMA codes. This schedule is announced by the BS during the setup time in each round and it is followed by the sensors during the steady state time. As a result of this schedule, a sensor (say sensor $\mathrm{x}$ ) waits until its determined time slot to report an event. During this waiting time, if other sensor reports the same event, then sensor $\mathrm{x}$ cancels the reporting process.

\subsection{Proposed time-scheduling algorithm}

The time-scheduling algorithm proposed can be explained using the example shown in Figure 5 as follows:

a. First, the BS assigns for each sensor in the network a unique time slot based on its energy; the first slot goes to the one with the most energy, and the final slot goes to the one with the least energy. Each sensor is assigned an index number (IN) which is the same as the time slot's index. It is clear that the length of the created time-schedule is directly proportional to the network density (i.e., number of nodes in the network). As a result, it may be very long. Accordingly, the next steps of this algorithm reduce the length of this schedule by allowing different sensors to share the same time slot. Assume that after applying this step on the example shown in Figure 5, the result is given Table 1:

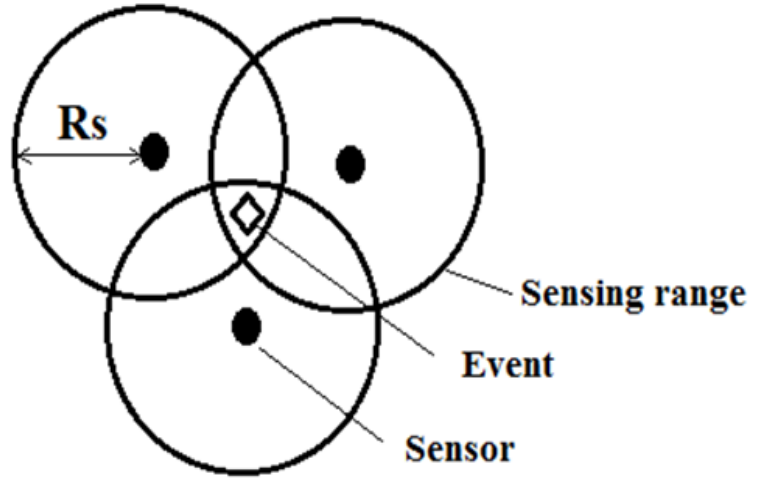

Figure 4. Illustration of the main idea behind SIICERP

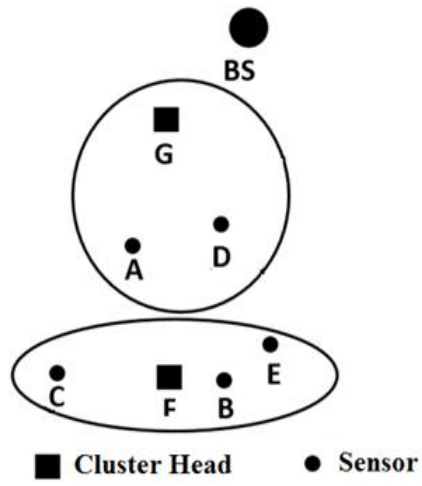

Figure 5. An example to illustrate the proposed time-scheduling algorithm 
Table 1. The result of applying the first step of the time-scheduling algorithm on the example shown in Figure 5

\begin{tabular}{ccl}
\hline Time-slot & IN & Sensor \\
\hline 1 & 1 & D \\
2 & 2 & F \\
3 & 3 & E \\
4 & 4 & C \\
5 & 5 & A \\
6 & 6 & G \\
7 & 7 & B \\
\hline
\end{tabular}

b. Second, starting with the sensor that has $\mathrm{IN}=1$, the INs of all its neighbors (i.e., that are located within the 2Rs range; see Table 2) are reset to 1 . This procedure is repeated to all sensors such that the IN (in this step) of any sensor is not changed more than once. The result of applying this step on the considered example is given in Table 3 .

Table 2. Neighbors of each sensor in the example shown in Figure 5

\begin{tabular}{ccc}
\hline Sensor & Neighbors & Cluster \\
\hline A & D & 1 \\
B & E, F & 2 \\
C & --- & 2 \\
D & A & 1 \\
E & B & 2 \\
F & B & 2 \\
G & --- & 1 \\
\hline
\end{tabular}

Table 3. The result of applying the second step of the time-scheduling algorithm on the example shown in Figure 5

\begin{tabular}{ccl}
\hline Time-slot & IN & Sensor \\
\hline 1 & 1 & D \\
2 & 2 & F \\
3 & 3 & E \\
4 & 4 & $\mathrm{C}$ \\
5 & 1 & A \\
6 & 6 & G \\
7 & 2 & B \\
\hline
\end{tabular}

c. Third, perform a new modification on the IN values based on the following two cases:

1) If a sensor's IN value equals the current time slot's index, then this indicates that the IN of this sensor was not changed in step 2 and no one of the time slots before the current time slot were assigned to one of its neighbors. Hence, it is possible to assign a new time slot (also new value for the IN) from the previous time slots if this new time slot was not assigned for another sensor from the same cluster. In this case, if the IN value of a sensor is reset to a new value, then the IN of all its neighbors should be changed to the new value.

2) If a sensor's IN value not equals the current time slot's index, then this indicates that the IN of this sensor was changed and the sensor has a neighbor in the time-slot of index IN and that neighbor has more energy. Hence, it is possible to assign a new time slot (also new value for the IN) from the prior time slots (starting with the one that has index of IN+1) if this new time slot was not assigned for another sensor from the same cluster. In this case, if the IN of a sensor is given a new value, then the INs of all its neighbors are changed to the new value.

The result of applying the third step on the considered example can be summarized as follows:

- The result after checking the IN of sensor F are shown in Table 4,

- The result after checking the IN of sensor E are shown in Table 5,

- The result after checking the IN of sensor $\mathrm{C}$ are shown in Table 6 ,

- The result after checking the IN of sensor A are shown in Table 7,

- The result after checking the IN of sensor G are shown in Table 8,

- The result after checking the IN of sensor B are shown in Table 9.

Based on the final schedule, each sensor listens to its neighbor during their time slots and sends its reports during its time slot. 
Table 4. The result of applying the third step (after checking the IN of sensor F) of the time-scheduling

\begin{tabular}{ccc}
\multicolumn{3}{c}{ algorithm on the example shown in Figure 5 } \\
\hline Time-slot & IN & Sensor \\
\hline 1 & 1 & D,F \\
2 & 2 & \\
3 & 3 & E \\
4 & 4 & C \\
5 & 1 & A \\
6 & 6 & G \\
7 & 1 & B \\
\hline
\end{tabular}

Table 5. The result of applying the third step (after checking the IN of sensor E) of the time-scheduling

\begin{tabular}{ccc}
\multicolumn{2}{c}{ algorithm on the example shown in Figure 5 } \\
\hline Time-slot & IN & Sensor \\
\hline 1 & 1 & D,F \\
2 & 2 & E \\
3 & 3 & \\
4 & 4 & $\mathrm{C}$ \\
5 & 1 & $\mathrm{~A}$ \\
6 & 6 & $\mathrm{G}$ \\
7 & 2 & $\mathrm{~B}$ \\
\hline
\end{tabular}

Table 6. The result of applying the third step (after checking the IN of sensor C) of the time-scheduling algorithm on the example shown in Figure 5

\begin{tabular}{ccc}
\hline Time-slot & IN & Sensor \\
\hline 1 & 1 & D,F \\
2 & 2 & E \\
3 & 3 & C \\
4 & 4 & \\
5 & 1 & A \\
6 & 6 & G \\
7 & 2 & B \\
\hline
\end{tabular}

Table 7. The result of applying the third step (after checking the IN of sensor A) of the time-scheduling

\begin{tabular}{ccc}
\multicolumn{3}{c}{ algorithm on the example shown in Figure 5 } \\
\hline Time-slot & IN & Sensor \\
\hline 1 & 1 & D,F \\
2 & 2 & E,A \\
3 & 3 & C \\
4 & 4 & \\
5 & 1 & \\
6 & 7 & G \\
7 & 2 & B \\
\hline
\end{tabular}

Table 8. The result of applying the third step (after checking the IN of sensor G) of the time-scheduling

\begin{tabular}{ccc}
\multicolumn{2}{c}{ algorithm on the example shown in Figure 5 } \\
\hline Time-slot & IN & Sensor \\
\hline 1 & 1 & D,F \\
2 & 2 & E,A \\
3 & 3 & C,G \\
4 & 4 & \\
5 & 1 & \\
6 & 7 & \\
7 & 2 & B \\
\hline
\end{tabular}

Table 9. The result of applying the third step (after checking the IN of sensor B) of the time-scheduling

\begin{tabular}{ccc}
\multicolumn{3}{c}{ algorithm on the example shown in Figure 5 } \\
\hline Time-slot & IN & Sensor \\
\hline 1 & 1 & D,F \\
2 & 2 & E,A \\
3 & 3 & C,G \\
4 & 4 & B \\
\hline
\end{tabular}




\section{PERFORMANCE EVALUATION}

Comprehensive simulations were run to assess SIICERP's performance. As we discussed before, SIICERP is a cluster-oriented routing protocol that can be used with any other cluster-oriented routing protocol. However, we select the two protocols that were discussed in section 2. One of them is called LEACH protocol [34] which is based on variable clustering (the clusters formation is changed with time) and the second one is called LBCH protocol [35] which is based on fixed clustering (the cluster formation is fixed through the lifetime of the network). In our simulations, we refer to LEACH and LBCH with SIICERP as SIICERP-LEACH and SIICERP-LBCH, respectively.

\subsection{Simulation parameters}

In our simulation, we consider different simulation scenarios. In Table 10, we summarize some of simulation parameters. These parameters were used in [34] and [35]. Other parameters will be specifically mentioned for each simulation scenario.

Table 10. Parameters for simulation

\begin{tabular}{cc}
\hline Parameter & Value \\
\hline Size of data packet & $1024 \mathrm{bits}$ \\
Size of control packet & $176 \mathrm{bits}$ \\
Prime energy & $2.4 \mathrm{~J}$ \\
Energy for transferring one bit (ETX) & $50 \mathrm{~nJ} / \mathrm{bit}$ \\
Energy for receiving one bit (ERX) & $50 \mathrm{~nJ} / \mathrm{bit}$ \\
Energy for data aggregation (EDA) & $5 \mathrm{~nJ} / \mathrm{bit}$ \\
Energy of transmit amplifier considering free & $10 \mathrm{PJ} / \mathrm{bit} / \mathrm{m}^{2}$ \\
space, propagation model & \\
Energy of transmit amplifier considering & $0.0013 \mathrm{PJ} / \mathrm{bit} / \mathrm{m}^{4}$ \\
multi-path, propagation model & \\
Simulation area & $100 \times 100 \mathrm{~m}$ \\
Sensors distribution & $\mathrm{Random}$ \\
Base-station position & $(50 \mathrm{~m}, 125 \mathrm{~m})$ \\
Sensing range & $10 \mathrm{~m}$ \\
\hline
\end{tabular}

\subsection{Simulation results}

\subsubsection{SIICERP-LEACH and LEACH protocols}

Figure 6 presents the effect of one part of IIER issue (i.e., sending redundant reporting packets from sensors to their $\mathrm{CHs}$ ). In this case, we run the simulation with 1000 rounds and one event is selected in a random position per round. The number of sensors in the network is changed and the mean number of CHs is chosen to be constant by selecting certain ratios of $\mathrm{CHs}(p)$ for each corresponding number of sensors. For example, for 100 sensors we set $\mathrm{p}=0.1$, for 300 sensors, we set $\mathrm{p}=0.034$, for 500 sensors, we set $\mathrm{p}=0.02$, and for 700 sensors, we set $p=0.0143$. Figure 6 indicates that by applying SIICERP with LEACH, the number of reporting packets sent to the $\mathrm{CHs}$ is limited to the number of events (i.e., 1000 events). This is because, with SIICERP, each event is reported by one packet only. However, without SIICERP, the issue of IIER will be shown clearly as the number of sensors over the network is increased; more sensors will redundantly send packets to report one event.

In Figure 7, we illustrate the effect of the other part of the IIER issue (i.e., sending redundant reporting packets from $\mathrm{CHs}$ to the BS to announce one event). To achieve this goal, we use 300 sensors with different number of CHs. We run this simulation for 1000 rounds and with one event created in a random position during each round. As we can see from Figure 7, SIICERP reduces the number of reporting packets sent from the CHs to the BS to the number of events (i.e., 1000 events). Without SIICRP, this number is increased with increasing the number of un-cooperated $\mathrm{CHs}$ that redundantly send many packets to report one event.

The results of a simulated scenario in which the proportion of $\mathrm{CHs}$ is 0.05 , the events' locations per round are dispersed over a grid shape, and the number of sensors is 100, are shown in Figures 8 and 9 bearing in mind that a single sensing range is considered as the shortest distance between events. This event allocation is selected so that each sensor senses the nearest event to it in each round and certainly has a packet to report it. Because the sensors are dispersed throughout the network, it's possible that various sensors detect the same event. It is clear from Figures 8 and 9 that SIICERP-LEACH achieves higher performance compared to LEACH protocol (i.e., $610 \%$ and $160 \%$ average improvements concerning the number of alive sensors and the total residual energy, respectively).

Figures 10 and 11 present the results of the simulation scenario used for Figures 8 and 9 except that in each round, a random number of events, which is uniformly distributed considering 1-50 interval, is chosen whereas their locations are spread randomly across the network area. From Figures 10 and 11, it is observed that 
the SIICERP improves the performance of LEACH protocol (i.e., $280 \%$ and $172 \%$ improvements regarding the number of alive sensors and the total residual energy, respectively).

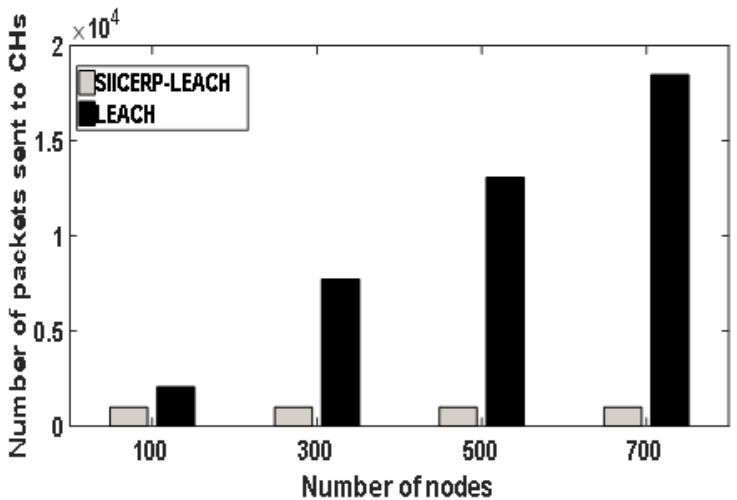

Figure 6. The number of reporting packets sent from the sensors to their CHs in the LEACH protocol with and without the proposed solution

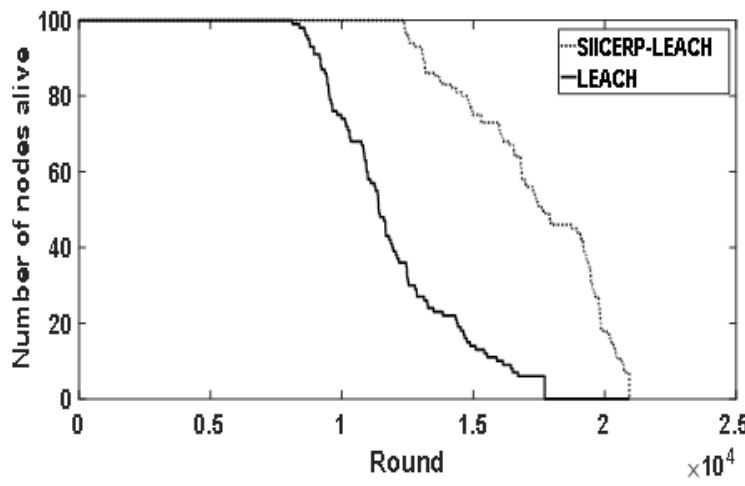

Figure 8. Number of sensors alive in the LEACH protocol with and without the proposed solution. Events locations have a grid distribution over the simulation area

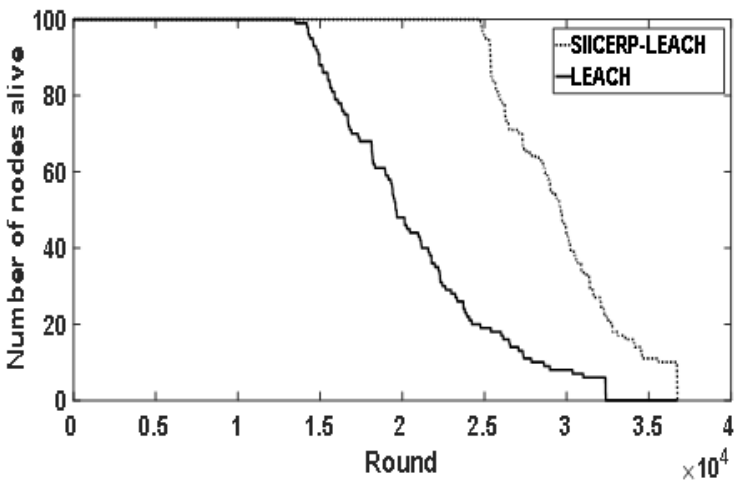

Figure 10. Number of sensors alive in the LEACH protocol with and without the proposed solution. Events locations have a random distribution over the simulation area

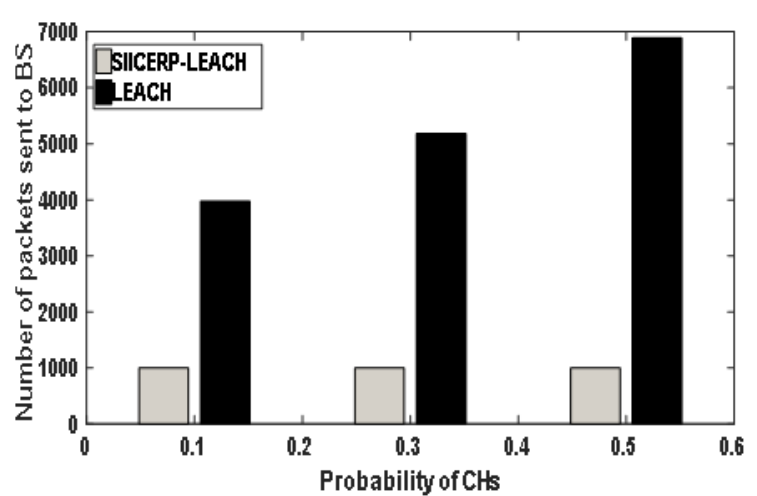

Figure 7. The number of reporting packets sent from the $\mathrm{CHs}$ to $\mathrm{BS}$ in the $\mathrm{LEACH}$ protocol with and without the proposed solution

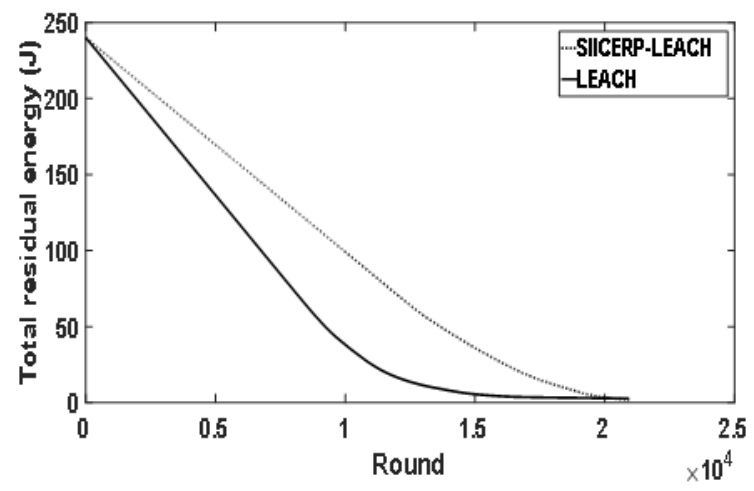

Figure 9. Total residual energy in the network in the LEACH protocol with and without the proposed solution. Events locations have a grid distribution over the simulation area

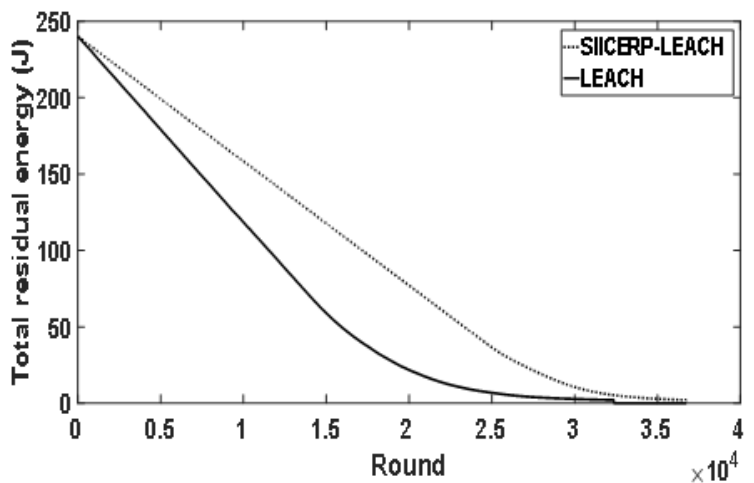

Figure 11. Total residual energy in the LEACH protocol with and without the proposed solution. Events locations have a random distribution over the simulation area 


\subsubsection{SIICERP-LBCH and $\mathrm{LBCH}$ protocols}

Since, in section 4.2.1, we thoroughly study the IIER problem. In this section, we just conduct two simulation scenarios to demonstrate the impact of SIICERP on LBCH performance. In both scenarios, the ratio of clusters is 0.05 . However, in one of them, the events' positions per round are located over a grid shape as discussed before in section 4.2.1. Figures 12 and 13 present the results of this simulation. In the other scenario, a random number of events (i.e., uniformly distributed in the range of 1 to 50) per round is selected and the locations of them are randomly distributed over the network area. Figures 14 and 15 depict the results of this scenario. From these figures, we can see that SIICERP effectively solves IIER issue.

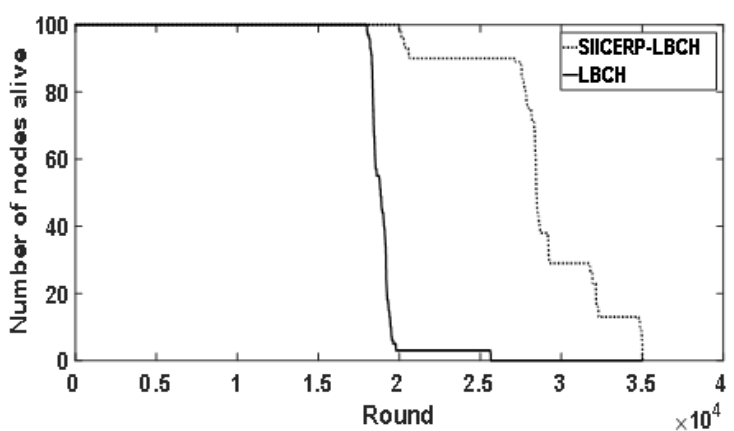

Figure 12. The total number of sensors alive in the $\mathrm{LBCH}$ protocol with and without the proposed solution. Events locations have a grid distribution over the simulation area

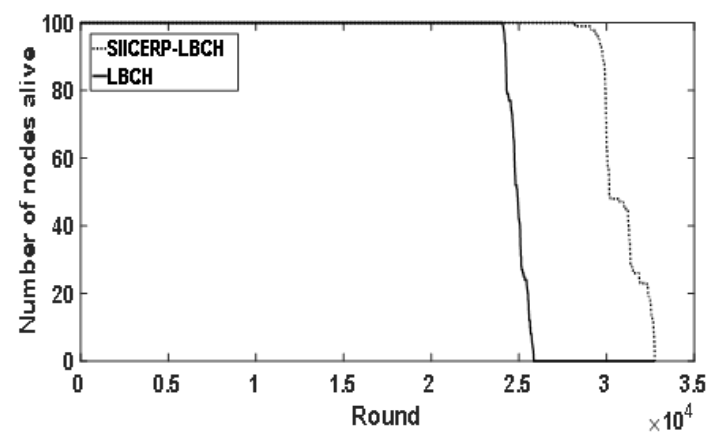

Figure 14. Number of sensors alive in the LBCH protocol with and without the proposed solution. Events locations have a random distribution over the simulation area

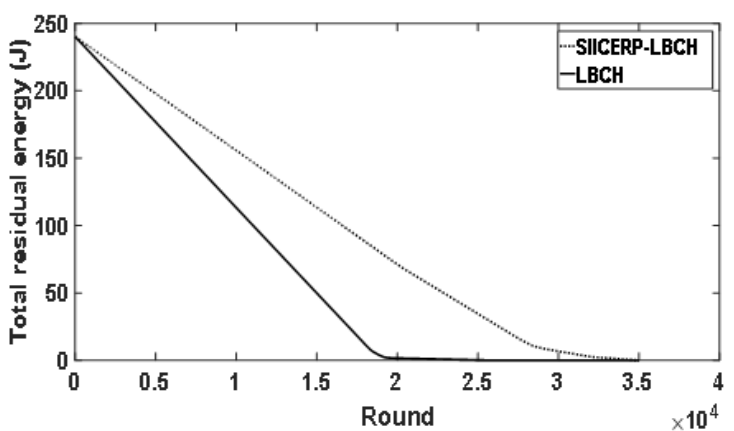

Figure 13. Total residual energy in the network in the $\mathrm{LBCH}$ protocol with and without the proposed solution. Events locations have a grid distribution over the simulation area

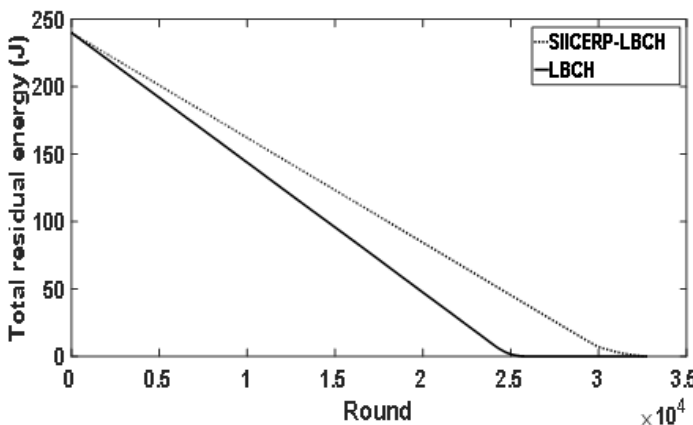

Figure 15. Total residual energy in the $\mathrm{LBCH}$ protocol with and without the proposed solution. Events locations have a random distribution over the simulation area

\section{CONCLUSION}

In this work, we studied an important issue in cluster-based routing schemes for WSNs; we found that many reporting packets are unnecessarily sent to the BS by the sensors and the CHs to report one event. Clearly, this will consume more energy in network. Therefore, we proposed a new solution for this issue. The new solution is basically based on generating a precise time-schedule, which will be received by all of the network's nodes. This time-schedule leads to reporting any event using a single packet sent by the sensor that senses this event and has the most remaining energy among all the sensors that sense the same event. Simulations show that the proposed solution enhances the cluster-oriented routing schemes' performance in the dimensions of load balancing and WSNs' lifetime.

\section{REFERENCES}

[1] S. Hong and K. Han, "Tree-based routing algorithms on wireless sensor networks: survey," Journal of Systems and Information Technology, vol. 16, no. 2, pp. 113-121, 2014, doi: 10.1108/JSIT-11-2013-0065.

[2] B. Gong and T. Jiang, "A tree-based routing protocol in wireless sensor networks," 2011 International Conference on Electrical and Control Engineering, Yichang, 2011, pp. 5729-5732, doi: 10.1109/ICECENG.2011.6057953. 
[3] M. H. Anisi, A. H. Abdullah, A. S. Razak, and M. A. Ngadi, "An overview of data routing approaches for wireless sensor networks," Sensors (Basel), vol. 12, no. 4, pp. 3964-3996, 2012, doi:10.3390/s120403964.

[4] A. Kanavalli, D. Sserubiri, P. D. Shenoy, K. R. Venugopal, and L. M. Patnaik, "A flat routing protocol for sensor networks," 2009 Proceeding of International Conference on Methods and Models in Computer Science (ICM2CS), Delhi, 2009, pp. 1-5, doi: 10.1109/ICM2CS.2009.5397948.

[5] H. Echoukairi, K. Bourgba, and M. Ouzzif, "A survey on flat routing protocols in wireless sensor networks," International Symposium on Ubiquitous Networking, vol. 366, 2015, pp. 311-324, doi: 10.1007/978-981-287-990-5_25.

[6] W. Dargie and J. Wen, "A simple clustering strategy for wireless sensor networks," IEEE Sensors Letters, vol. 4, no. 6, pp. 1-4, 2020, Art no. 7500804, doi: 10.1109/LSENS.2020.2991221.

[7] R. T. Al-Zubi, M. Q. Alawad, A. A. Kreishan and K. A. Darabkh, "A yet efficient event reporting protocol for wireless sensor networks," 2021 IEEE International IOT, Electronics and Mechatronics Conference (IEMTRONICS), Toronto, Canada, 2021, pp. 1-5, doi: 10.1109/IEMTRONICS52119.2021.9422558.

[8] M. Mirzaie and S. M. Mazinani, "AFLCH: Self-adaptive unequal fuzzy based clustering of heterogeneous sensors in wireless sensor networks,” 2020 28th Iranian Conf. on Electrical Engineering (ICEE), 2020, pp. 1-5, doi: 10.1109/ICEE50131.2020.9260624.

[9] K. A. Darabkh, E. N. AL-zoubi, F. A. Al-naimat, and A. F. Khalifeh, "Mobile sink optimization for enhancing data delivery in wireless sensor networks," 2020 IEEE International IOT, Electronics and Mechatronics Conference (IEMTRONICS), Vancouver, Canada, 2020, pp. 1-4, doi: 10.1109/IEMTRONICS51293.2020.9216356.

[10] B. Kumar, U. K. Tiwari, and S. Kumar, "Energy efficient quad clustering based on k-means algorithm for wireless sensor network," 2020 Sixth International Conference on Parallel, Distributed and Grid Computing (PDGC), 2020, pp. 73-77, doi: 10.1109/PDGC50313.2020.9315853.

[11] P. K. Mishra and S. K. Verma, "A survey on clustering in wireless sensor network," 2020 11th International Conference on Computing, Communication and Networking Technologies (ICCCNT), 2020, pp. 1-5, doi: 10.1109/ICCCNT49239.2020.9225420.

[12] A. F. Khalifeh, H. Abid, and K. A. Darabkh, "Double Mobility WSN: exploiting the mobility of sink and cluster head nodes for better WSN energy preservation and lifetime," 2020 IEEE International IOT, Electronics and Mechatronics Conference (IEMTRONICS), Vancouver, Canada, 2020, pp. 1-4, doi: 10.1109/IEMTRONICS51293.2020.9216449.

[13] L. Chen, W. Liu, D. Gong, and Y. Chen, "Cluster-based routing algorithm for WSN based on subtractive clustering," 2020 Int. Wireless Communications and Mobile Computing (IWCMC), 2020, pp. 403-406, doi: 10.1109/IWCMC48107.2020.9148244.

[14] K. A. Darabkh, W. Kassab, and A. Khalifeh, "Maximizing the life time of wireless sensor networks over iot environment," 2020 Fifth International Conference on Fog and Mobile Edge Computing (FMEC), Paris, France, 2020, pp. 270-274, doi: 10.1109/FMEC49853.2020.9144826.

[15] P. K. Mishra and S. K. Verma, "OCSSP: Optimal cluster size selection-based clustering protocol using fuzzy logic for wireless sensor network," 2020 IEEE International Conference on Advent Trends in Multidisciplinary Research and Innovation (ICATMRI), 2020, pp. 1-4, doi: 10.1109/ICATMRI51801.2020.9398435.

[16] K. A. Darabkh, J. Zomot, Z. Al-qudah, and A. Khalifeh, "IEDB-CHS-BOF: Improved energy and distance based CH selection with balanced objective function for wireless sensor networks," 2020 Fifth International Conference on Fog and Mobile Edge Computing (FMEC), Paris, France, 2020, pp. 275-279, doi: 10.1109/FMEC49853.2020.9144808.

[17] Z. Hammodi, A. A. Hilli, and M. Al-Ibadi, "Optimal placement of single cluster head in wireless sensor networks via clustering," 2021 IEEE 11th Annual Computing and Communication Workshop and Conference (CCWC), 2021, pp. 1157-1160, doi: 10.1109/CCWC51732.2021.9376150.

[18] A. Khalifeh et al., "Wireless sensor networks for smart cities: Network design, implementation and performance evaluation," Electronics, vol. 10, no. 2, 2021, Art. No. 218, doi: 10.3390/electronics10020218.

[19] T. J. Swamy, G. Ramamurthy, and P. Nayak, "Optimal, secure cluster head placement through source coding techniques in wireless sensor networks," IEEE Communications Letters, vol. 24, no. 2, pp. 443-446, 2020, doi: 10.1109/LCOMM.2019.2953850.

[20] B. Zhu, E. Bedeer, H. H. Nguyen, R. Barton, and J. Henry, "Improved soft-k-means clustering algorithm for balancing energy consumption in wireless sensor networks," IEEE Internet of Things Journal, vol. 8, no. 6, pp. 4868-4881, 2021, doi: $10.1109 /$ JIOT.2020.3031272.

[21] S. Umbreen, D. Shehzad, N. Shafi, B. Khan, and U. Habib, “An energy-efficient mobility-based cluster head selection for lifetime enhancement of wireless sensor networks,” IEEE Access, vol. 8, pp. 207779-207793, 2020, doi: 10.1109/ACCESS.2020.3038031.

[22] Y. Tao, J. Zhang, and L. Yang, "An unequal clustering algorithm for wireless sensor networks based on interval type-2 TSK fuzzy logic theory," IEEE Access, vol. 8, pp. 197173-197183, 2020, doi: 10.1109/ACCESS.2020.3034607.

[23] A. Khalifeh et al., "Radio frequency based wireless charging for unsupervised clustered WSN: System implementation and experimental evaluation," Energies, vol. 14, no. 7, pp. 1-21, 2021, doi: 10.3390/en14071829.

[24] T. Zhang, G. Chen, Q. Zeng, G. Song, C. Li, and H. Duan, "Routing clustering protocol for 3D wireless sensor networks based on fragile collection ant colony algorithm," IEEE Access, vol. 8, pp. 58874-58888, 2020, doi: 10.1109/ACCESS.2020.2982691.

[25] Wafa'a Kassab and Khalid A. Darabkh, "A-Z survey of internet of things: Architectures, protocols, applications, recent advances, future directions and recommendations," Journal of Network and Computer Applications, vol. 163, 2020, Art. no. 102663, doi: 10.1016/j.jnca.2020.102663.

[26] F. Lin, W. Dai, W. Li, Z. Xu, and L. Yuan, "A framework of priority-aware packet transmission scheduling in cluster-based industrial wireless sensor networks," IEEE Transactions on Industrial Informatics, vol. 16, no. 8, pp. 5596-5606, 2020, doi: 10.1109/TII.2019.2944980.

[27] K. A. Darabkh, J. N. Zomot, and Z. Al-qudah, "EDB-CHS-BOF: Energy and distance based cluster head selection with balanced objective function protocol," IET Communications, vo. 13, no. 19, pp. 3168-3180, 2019, doi: 10.1049/iet-com.2019.0092.

[28] A. Arulmurugan, S. F. Waris, and N. Bhagyalakshmi, "Analysis of cluster head selection methods In WSN," 2021 6th International Conference on Inventive Computation Technologies (ICICT), 2021, pp. 114-119, doi: 10.1109/ICICT50816.2021.9358532.

[29] K. A. Darabkh, W. S. Al-Rawashdeh, R. T. Al-Zubi, and S. H. Alnabelsi, "C-DTB-CHR: Centralized density and threshold-based cluster head replacement protocols for wireless sensor networks," The Journal of Supercomputing, vol. 73, no. 12, pp. 5332-5353, 2017, doi: 10.1007/s11227-017-2089-4.

[30] B. Pitchaimanickam, "Dragonfly algorithm for hierarchical clustering in wireless sensor networks," 5th International Conference on Intelligent Computing and Control Systems (ICICCS), 2021, pp. 192-197, doi: 10.1109/ICICCS51141.2021.9432337.

[31] R. T. Ai-Zubi, N. Abedsalam, A. Atieh and K. A. Darabkh, "Lifetime-improvement routing protocol for wireless sensor networks," 2018 15th International Multi-Conference on Systems, Signals \& Devices (SSD), Hammamet, Tunisia, 2018, pp. 683-687, doi: 10.1109/SSD.2018.8570628

[32] H. Amirinia and R. Liscano, "Optimized application driven scheduling for clustered WSN," 2020 International Symposium on Networks, Computers and Communications (ISNCC), 2020, pp. 1-6, doi: 10.1109/ISNCC49221.2020.9297230 
[33] R. T. Al-Zubi, A. A. Kreishan, M. Q. Alawad and K. A. Darabkh, "On the event reporting of intra/inter-cluster sensor networks," 2021 IEEE International IOT, Electronics and Mechatronics Conference (IEMTRONICS), Toronto, Canada, 2021, pp. 1-6, doi: 10.1109/IEMTRONICS52119.2021.9422549.

[34] W. R. Heinzelman, A. Chandrakasan, and H. Balakrishnan, "Energy-efficient communication protocol for wireless microsensor networks," Proceedings of the 33rd Hawaii International Conference on System Sciences, 2000, doi: 10.1109/HICSS.2000.926982.

[35] R. T. Al-Zubi, N. Abedsalam, A. Atieh, and K. A. Darabkh, "LBCH: Load balancing cluster head protocol for wireless sensor networks," Informatica, vol. 29, no. 4, pp. 633-650, 2018

[36] A. More and V. Raisinghani, "A survey on energy efficient coverage protocols in wireless sensor networks," Journal of King Saud University-Computer and Information Sciences, vol. 29, no. 4, pp. 428-448, 2017, doi: 10.1016/j.jksuci.2016.08.001.

\section{BIOGRAPHIES OF AUTHORS}
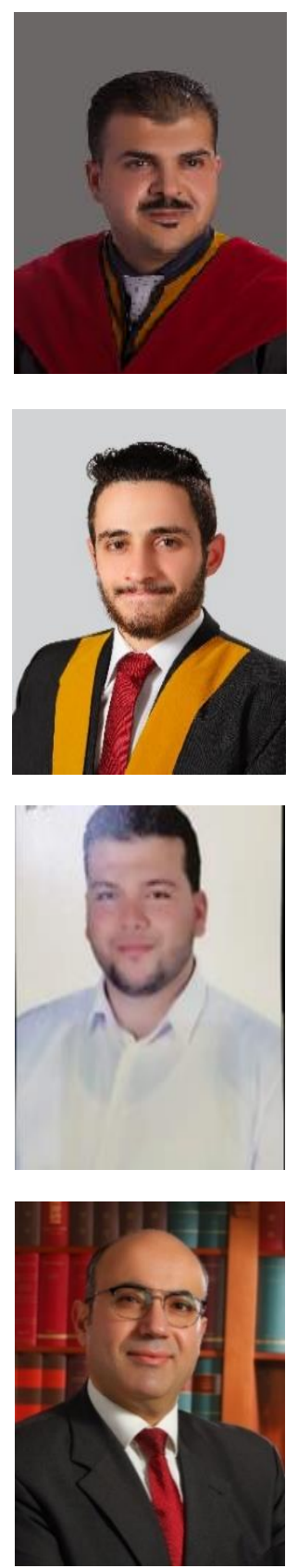

Raed Taleb Al-Zubi (D) 8 SC $\mathrm{P}$ received the PhD degree in Electrical and Computer Engineering/Wireless communications and Networking from University of Arizona-USA, in 2010. His PhD thesis was under supervision of Prof. Marwan Krunz. Prof. Al-Zubi was granted a full scholarship from the University of Jordan-Jordan to get his $\mathrm{PhD}$ degree. $\mathrm{He}$ received BS and MS degrees in Electrical Engineering from the university of Jordan- Jordan, in 2002 and 2005, respectively. He is currently a full professor at the department of electrical engineering in the University of Jordan. His current research interests include system architecture, communication protocol designs for wireless networks with emphasis on crosslayer designs for ultra-wideband (UWB) networks, wireless sensor networks, cognitive radio networks, biometrics, pattern recognition, optical communications, and image processing. $\mathrm{He}$ can be contacted at email: r.alzubi@ju.edu.jo.

Abdulraheem Ahmed Kreishan (iD SO SC received the BS degree in Electrical Engineering from the University of Jordan-Jordan, in 2020. His graduation project was under supervision of Prof. Raed Al-Zubi, with a title of "Energy Efficient Data Collection Protocol for Wireless Sensor Networks". He is currently a site engineer at Jordan School Expansion Project (JSEP) funded by USAID. His current research interests include research \& development, communication protocol designs for wireless networks with emphasis on routing and limitations issues, wireless sensor networks, networks and routers configurations, CCNA, and renewable energy resources designing and installation. He can be contacted at email: abdulraheem.kreishan97@gmail.com.

Mohammad Qasem Alawad (D) SI SC P received the BS degree in Electrical Engineering from the university of Jordan- Jordan, in 2020. His current research interests focus on wireless sensor networks. He can be contacted at email: m.qasem@bk.ru.

Khalid Ahmad Darabkh (D) SC $\mathrm{P}$ received the $\mathrm{PhD}$ degree in Computer Engineering from the University of Alabama in Huntsville (UAH), USA, in 2007 with honors. He was selected for inclusion in the Who's Who Among Students in American Universities and Colleges. He was honored by UAH for being not only so outstanding, but also finishing the $\mathrm{PhD}$ requirements along with publishing a massive number of reputable scientific articles in record time. He has joined the Computer Engineering Department at the University of Jordan as an Assistant Professor since 2007 and obtained a distinguished professorship in 2016. He authored and co-authored of at least a hundred seventy highly esteemed research articles. $\mathrm{He}$ is among World's Top 2\% Scientists List, for various disciplines, compiled by Stanford University in 2020. Prof. Darabkh is the recipient of 2020 Federation of Arab Scientific Research Councils Reward-Theme of Innovation. Prof. Darabkh is further the recipient of Ali Mango Distinguished Researcher Reward for Scientific Colleges and Research Centers in Jordan. He is among Top 5 JU Researcher's List who published the highest number of quality manuscripts in Scopus. He is engaged in research mainly on Internet of things, Softwaredefined networks, vehicular networks, flying ad-hoc networks, Fog networking, Full duplex cognitive radio networks, queuing systems and networks, multimedia transmission, channel coding, steganography and watermarking, as well as innovative and interactive learning environments. He can be contacted at email: k.darabkeh@ju.edu.jo. 